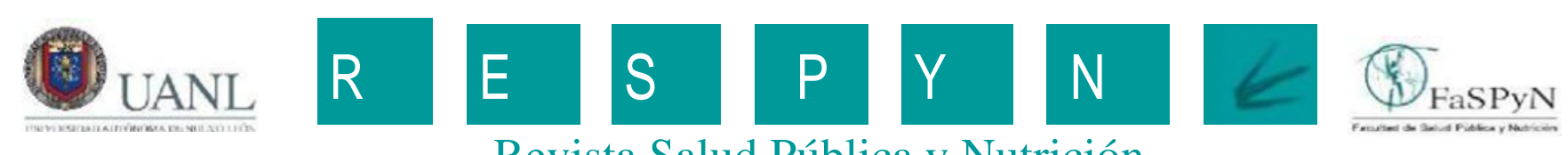

Revista Salud Pública y Nutrición

\title{
IMPACTO DEL BAJO CONSUMO DE ALIMENTOS CON GLUTEN Y CASEÍNA SOBRE SÍNTOMAS GASTROINTESTINALES EN NIÑOS DE 3-12 AÑOS DE EDAD CONTRASTORNO DEL ESPECTRO AUTISTA.
}

IMPACT OF LOW FOOD CONSUMPTION WITH GLUTEN AND CASEINE ON GASTROINTESTINAL SYMPTOMS IN CHILDREN 3-12 YEARS OLD WITH AUTISM SPECTRUM DISORDER

Hernández Cruz Ana Cristina*, López Ordaz Lyleni Guadalupe*, Garza Sepúlveda Gerardo*, Cuellar Robles Sofía*, Márquez Zamora Leticia*, Sánchez Peña María Alejandra*

*Universidad Autónoma de Nuevo León (UANL), México.

Citation: Hernández Cruz AC., López Ordaz LG., Garza Sepúlveda G., Cuellar Robles S., Márquez Zamora L., Sánchez Peña MA. (2017) Impacto del bajo consumo de alimentos con gluten y caseína sobre síntomas gastrointestinales en niños de 3-12 años de edad con trastorno del espectro autista. Revista de Salud Pública y Nutrición, 16(3), 1-5.

Editor: Esteban G. Ramos Peña, Dr. CS., Universidad Autónoma de Nuevo león, Facultad de Salud Pública, Monterrey Nuevo León, México.

Copyright: @2017 Hernández Cruz AC et al. This is an open-access article distributed under the terms of Creative Commons Attribution License [CC BY-ND 4.0], which permits unrestricted use, distribution, and reproduction in any medium, provided the original author and source are credited.

Competing interests: The authors have declared that no competing interests exist.

DOI: https://doi.org/10.29105/respyn16.3-1

Email: maria.sanchezp@uanl.mx 


\title{
IMPACTO DEL BAJO CONSUMO DE ALIMENTOS CON GLUTEN Y CASEÍNA SOBRE SÍNTOMAS GASTROINTESTINALES EN NIÑOS DE 3-12 AÑOS DE EDAD CON TRASTORNO DEL ESPECTRO AUTISTA
}

\author{
Hernández Cruz Ana Cristina*, López Ordaz Lyleni Guadalupe*, Garza Sepúlveda Gerardo*, Cuellar Robles \\ Sofía*, Márquez Zamora Leticia*, Sánchez Peña María Alejandra*
}

*Universidad Autónoma de Nuevo León (UANL), México

\begin{abstract}
RESUMEN
Introducción: Los trastornos del espectro autista, presentan alteraciones gastrointestinales tales como diarrea, flatulencia, reflujo gastroesofágico y constipación las cuales se han relacionado con el consumo de dietas con gluten y caseína. Objetivo: Evaluar el efecto de la dieta sin gluten y caseína sobre síntomas gastrointestinales en niños con trastornos del espectro autista. Material y Métodos: Previo consentimiento del padre o tutor se evaluaron niños de 3 a 12 años de edad, aplicando una historia nutricional con datos antropométricos y dietéticos por frecuencia alimentaria; la permeabilidad intestinal se evaluó por el cuestionario de signos y síntomas gastrointestinales "brea King the vicious cycle", intestinal health trhough diet, al inicio y final de la intervención durante 10 semanas. Donde se proporcionó menú semanal de acuerdo a recomendaciones calóricas por edad con alimentos libres de gluten y caseína. Los datos se analizaron por estadística descriptiva y pruebas no paramétricas utilizando $\mathrm{Chi}^{2}$ de dos variables, test de McNemar y prueba de Friedman utilizando el paquete estadístico SPSS versión 24.0. Resultados: Del total de 26 sujetos, $77 \%$ de género masculino y $23 \%$ femenino, con media de edad $7.6+2.6$ años, se observó una disminución del consumo de alimentos con gluten y caseína a menos de tres veces por semana, lo cual aminora la presencia de distención abdominal, diarrea, flatulencia. Siendo la permeabilidad intestinal estadísticamente significativa $(\leq 0.05)$ después de 10 semanas de intervención. Conclusiones: La disminución en el consumo de alimentos con gluten y caseína disminuye síntomas gastrointestinales asociados a permeabilidad intestinal.

Palabras Clave: Autismo, nutrición, gluten, caseína.
\end{abstract}

\section{ABSTRACT}

Introduction: Autistic spectrum disorders present gastrointestinal disorders such as diarrhea, flatulence, gastroesophageal reflux and constipation, which have been related to the consumption of diets with gluten and casein. Objective: To evaluate the effect of the gluten-free and casein-free diet on gastrointestinal symptoms in children with autism spectrum disorders. Methods: After the consent of the parent or guardian, children 3 to 12 years of age were evaluated, applying a nutritional history with anthropometric and dietary data by food frequency; intestinal permeability was assessed by the questionnaire for gastrointestinal signs and symptoms "breaking the vicious cycle", intestinal health through diet, at the beginning and end of the intervention for 10 weeks. Where weekly menu was provided according to calorie-age recommendations with gluten-free and casein-free foods. Data were analyzed by descriptive statistics and non-parametric tests using Chi2 of two variables, McNemar test and Friedman test using the statistical package SPSS version. Results Of the total of 26 subjects, $77 \%$ male and $23 \%$ female, with a mean age of $7.6+2.6$ years, a decrease in food consumption with gluten and casein was observed less than three times a week, The presence of abdominal distention, diarrhea, flatulence. The intestinal permeability was statistically significant $(\leq 0.05)$ after 10 weeks of intervention. Conclusions: The decrease in food consumption with gluten and casein decreases gastrointestinal symptoms associated with intestinal permeability

Key words: Autism, nutrition, gluten, casein 


\section{Introducción}

Los trastornos del espectro autista (TEA) también denominados trastornos generalizados del desarrollo, son un conjunto de problemas vinculados al neurodesarrollo con manifestaciones cognitivas y de comportamiento que ocasionan notables limitaciones en la autonomía personal. (CabanyesGarcía, 2004), de acuerdo al Centers for Disease Control and Prevention (CDC) en 2014, uno de cada 68 niños ha sido identificado con TEA; aconteciendo en todas las razas, etnias y grupos socioeconómicos, con prevalencia de 4.5 veces más común en niños (1 de 42) que en niñas (1 de 189). Esta población es propensa a desarrollar alergias alimentarias, intolerancias e hipersensibilidad a diversos alimentos y derivados como el gluten y caseína y a colorantes, los cuales provocan una serie de alteraciones gastrointestinales, cutáneas y fiebres inexplicables, que puede afectar su comportamiento, la causa de esta sintomatología aún no está clara, pero se relaciona con una flora intestinal anormal y un posible exceso de uso de antibióticos que puede lesionarla (Elder, J. H. et al, 2006) (Adams B. J. et al. 2011).

La terapia dietética en esta población es de importancia ya que desde hace años diversos estudios han relacionado algunas restricciones en la dieta y su efecto en el comportamiento de los niños con TEA sobre todo de gluten y caseína, con la teoría particular de que no pueden ser digeridas por completo, generando péptidos que pueden actuar como opiáceos; aparentemente por una deficiencia enzimática la cual impide desdoblar adecuadamente las proteínas. Como resultado de esto, surgen dos sustancias derivadas de la digestión incompleta de las proteínas del gluten y la caseína, la gluteomorfina y la caseomorfina, ambos péptidos bioactivos pueden atravesar la membrana intestinal y llegar a tejidos periféricos vía circulación sistémica, logrando alterar el metabolismo celular como los inductores hormonales y neurotransmisores (Audisio A. et al, 2013).

Por lo anterior la dieta libre de gluten y caseína se considera una intervención eficaz para mejorar los síntomas característicos del TEA, obteniendo disminución de la hiperactividad, y los problemas gastrointestinales (GI) que incluyen diarrea, constipación, distensión, dolor abdominal, pirosis, heces fétidas, presencia de moco, sangre o con restos alimentarios, esteatorrea, flatulencia, eructos y reflujo gastroesofágico (Villatoro $\mathrm{M}$. et al, 2015) (Toh C., M. y Allen V. E., 2015) ( Harris C. and Card B. 2012). Debido a esto, el objetivo de este estudio Evaluar el efecto de una dieta sin gluten y caseína que permita disminuir los signos y síntomas gastrointestinales en los niños con diagnóstico de TEA.

\section{Material y Métodos}

Estudio cuasi experimental, descriptivo, no aleatorio, con muestra autoseleccionada incluyendo niños ambos géneros de 3 a 12 años de edad con diagnóstico establecido de TEA por un especialista del neurodesarrollo, a los cuales bajo previo consentimiento del padre o tutor se aplicó por profesionales de la salud una evaluación al inicio y final de la intervención de 10 semanas, utilizando las técnicas de medición establecidas (OMS, 1995) para la historia nutricional los datos antropométricos de peso por medio de una báscula seca modelo 874 , la estatura determinada con estadímetro seca modelo 213 e índice de masa corporal (IMC) $\mathrm{kg} / \mathrm{m}^{2}$, interpretado de acuerdo a tablas de IMC para la edad de OMS 2007. Para los datos dietéticos se utilizó la frecuencia alimentaria la cual mostraba alimentos con gluten y caseína con la finalidad de evaluar si el consumo de estos alimentos era menor o mayor a tres veces por semana, ya que la frecuencia mayor a 3 veces agrava los síntomas gastrointestinales. La permeabilidad intestinal se determinó por el cuestionario de signos y síntomas gastrointestinales "breaking the vicious cycle", intestinal health trhough diet, evaluada de acuerdo al puntaje: 1-5 puntos: permeabilidad intestinal menos propensa a presentarse como categoría 1, categoría 2 de 6-10 puntos: posiblemente puede presentar permeabilidad intestinal, categoría 3 de 11-19 puntos: permeabilidad intestinal posiblemente presente, de 20 o más puntos: permeabilidad intestinal тиу posiblemente presente como categoría 4 (Gottschall E., 2004), ( Lázaro C. P. et al. 2016), ya que una gran mayoría de sujetos con TEA sufren problemas gastrointestinales lo que los lleva a una anormalidad en la mucosa intestinal asociada con una permeabilidad selectiva (Salles T. T., E. et al. 2014,).

Posterior a la recolección de datos, se entregó plan de alimentación individualizado por menús con requerimientos para la edad según la OMS 2001, 
libre de caseína y gluten, donde las distribuciones de macronutrientes fueron de $53 \%$ de hidratos de carbono, $14 \%$ de proteína y $33 \%$ de grasas para los grupos de 3 a 7 años de edad y $50 \%$ de hidratos de carbono, $20 \%$ de proteína y $30 \%$ de grasas para los grupos de 8 a 12 años de edad, realizado por 2 licenciadas en nutrición estudiantes de la especialidad en nutriología clínica de la Facultad de Salud Pública y Nutrición de la Universidad Autónoma de Nuevo León, posteriormente a las 10 semanas de intervención se realizaron nuevamente las evaluaciones.

Los datos fueron analizados por el paquete estadístico SPSS versión 24.0, realizando estadística no paramétrica, descriptiva y comparativa con un nivel de significancia de $\mathrm{p}<0.05$ e intervalo de confianza del $95 \%$, utilizando test de McNemar para evaluar el cambio en la frecuencia dietética, $\mathrm{Chi}^{2}$ para relacionar el consumo de alimentos con la sintomatología y la prueba de Friedman, para comparar los cambios en el cuestionario de signos y síntomas.

\section{Resultados}

Se evaluó un total de 26 niños, siendo el $77 \%(n=20)$ de género masculino y $23 \%(n=6)$ femeninos, con edad promedio de $7.6 \pm 2.6$ años, en relación al diagnóstico médico un $58 \% \quad(\mathrm{n}=15)$ presentaron Autismo y $42 \%(\mathrm{n}=11)$ Asperger.

El estado nutricional de la población estudiada por índice de masa corporal para la edad (IMC/edad) el $54 \%$ se encuentra normal con peso saludable para la edad como se aprecia en la Figura1.

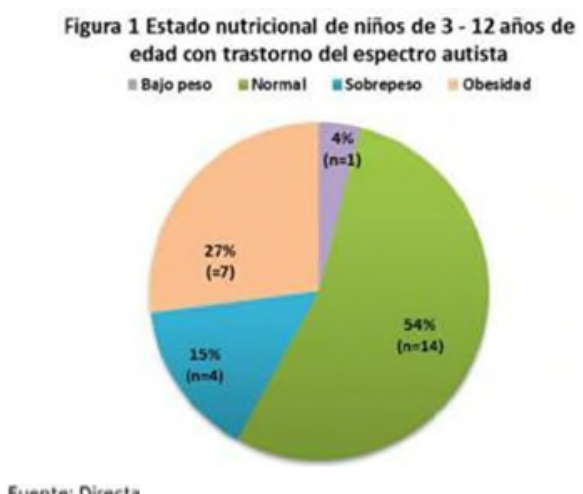

La evaluación de la frecuencia alimentaria se observa en la Tabla 1, siendo significativa ( $\mathrm{p}<0.05$ ) la disminución en el consumo de alimentos con gluten como tortillas de harina, pan de barra, pastas, pan dulce y galletas, y en caseína como leche y queso.

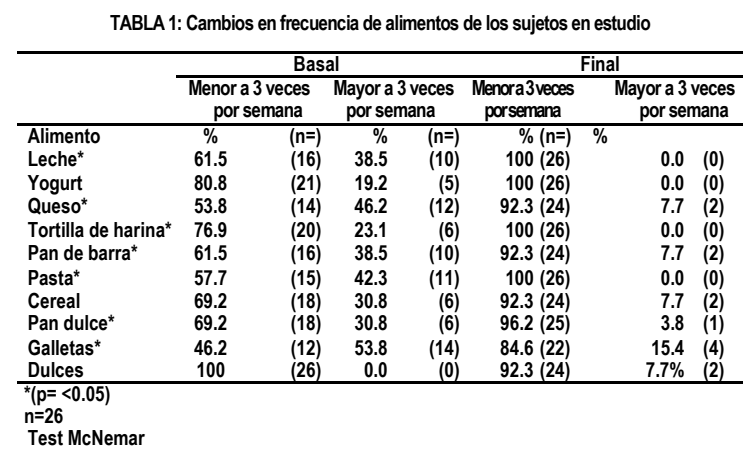

La evaluación de permeabilidad intestinal de acuerdo a los criterios de "breaking the vicious cycle", intestinal health trhough diet, se observan en la Figura 2 y tabla 2, donde se observó un cambio estadísticamente significativo ( $\mathrm{p}<0.05)$, ya que en la evaluación inicial el $96 \%(n=25)$ de los niños presentaba permeabilidad intestinal en la categoría 2 y 3 y al finalizar la intervención en la categoría 1 "menos propensa a presentarse" aumento de 4\% (1) a $31 \%(8)$, y en categoría 2 "puede presentar permeabilidad intestinal" de $39 \%$ (10) a $42 \%$ (11), observándose la mayor disminución en la categoría 3 "permeabilidad intestinal posiblemente presente" donde al inicio se manifestó un 57\% (15) y al final únicamente el $23 \%(\mathrm{n}=6)$ lo presentaron, para la categoría 4 "permeabilidad intestinal mиy posiblemente presente" al inicio no se observó y posterior al finalizar el tratamiento el $4 \%$ (1) lo manifestó de acuerdo a lo comparado por la prueba de Friedman analizando los signos y síntomas de permeabilidad intestinal al inicio $y$ al final al disminuir su consumo de leche, yogurt y pan de barra. 
TABLA 2: Cambios de signos y síntomas gastrointestinales en los sujetos en estudio

\begin{tabular}{|c|c|c|c|c|c|c|c|}
\hline & \multicolumn{3}{|c|}{ Basal } & \multicolumn{4}{|c|}{ Final } \\
\hline & No & Si & & & No & $\mathrm{Si}$ & \\
\hline Síntoma & $\%$ (n=) & $\%$ & $(n=)$ & $\%$ & $(n=)$ & $\%$ & $\begin{array}{c}(n=) \\
(3)\end{array}$ \\
\hline $\begin{array}{l}\text { Pirosis } \\
\text { Meteorismo }\end{array}$ & $\begin{array}{l}73.1 \text { (19) } \\
73.1 \text { (19) }\end{array}$ & $\begin{array}{l}26.9 \\
26.9\end{array}$ & $\begin{array}{l}(1) \\
(7)\end{array}$ & $\begin{array}{l}88.5 \\
69.2\end{array}$ & $\begin{array}{l}(23) \\
(18)\end{array}$ & $\begin{array}{l}11.5 \\
30.8\end{array}$ & $\begin{array}{l}\text { (3) } \\
\text { (8) }\end{array}$ \\
\hline Distención & 53.8 (14) & 46.2 & (12) & 73.1 & (19) & 26.9 & (7) \\
\hline Flatulencias * & 38.5 (10) & 61.5 & (16) & 61.5 & (16) & 38.5 & (10) \\
\hline Estreñimiento * & 50.0 (13) & 50.0 & (13) & 84.6 & (22) & 15.4 & (4) \\
\hline Diarrea & $80.8(21)$ & 19.3 & (5) & 96.2 & (25) & 3.8 & (1) \\
\hline Gastritis & $80.8(21)$ & 19.2 & (5) & 92.3 & (24) & 7.7 & (2) \\
\hline Colitis & 84.6 (22) & 15.4 & (4) & 92.3 & (24) & 7.7 & (2) \\
\hline Halitosis & 57.7 (15) & 42.3 & (11) & 76.9 & (20) & 23.1 & (6) \\
\hline Nicturia & $92.3(24)$ & 7.7 & (2) & 84.6 & (22) & 15.4 & (4) \\
\hline Poliuria & $80.8(21)$ & 19.2 & (5) & 65.4 & (17) & 34.6 & (9) \\
\hline Insomnio & $76.9(20)$ & 23.1 & (6) & 84.6 & (22) & 15.4 & (4) \\
\hline Rash & $92.3(24)$ & 7.7 & (2) & 100 & (26) & 0.0 & (0) \\
\hline Dermatitis & 84.6 (22) & 15.4 & (4) & 80.8 & (21) & 19.2 & (5) \\
\hline
\end{tabular}

Figura 2. Evaluación inicial y final de la permeabilidad intestinal

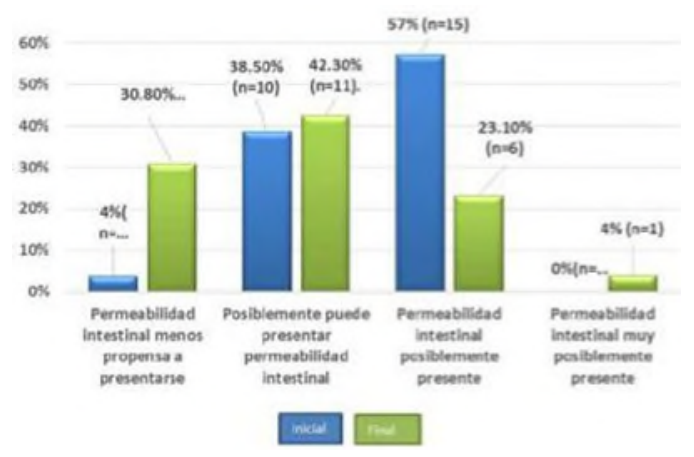

\section{Discusión}

El estado nutricional de nuestra población en estudio se mantuvo dentro del diagnóstico establecido al inicio del mismo, lo que sugiere el apego a su intervención nutricional.

También se pudo observar disminución a menos de 3 veces por semana del consumo de alimentos con gluten y caseína con la sintomatología intestinal como lo reportado por Pennesi M. C. y Cousino K. L en 2012, que sugieren que la DLGC es más efectiva en niños con trastorno del espectro autista con historia clínica de signos y síntomas gastrointestinales o en aquellos con sospecha de sensibilidad a algunos alimentos.
Dentro de los signos y síntomas gastrointestinales en nuestro estudio disminuyeron la distensión, diarrea y flatulencias como lo reportado por Ghalichi F. et al, 2016, quien revelo diferencia significativa en la disminución de dolor de estómago, inflamación, estreñimiento y diarrea después de llevar a cabo la DLGC, lo cual coincide con los estudios de Horvath K., y Perman J. en el 2002.

El tiempo de intervención nutricional fue de un lapso de 10 semanas, a diferencia otros estudios que muestran resultados en un lapso mayor a 6 meses como Pennesi M. C. y Cousino K. C., 2012, otros como el de Pedersen et al en el 2012 sugieren mayor probabilidad de responder a una dieta con un seguimiento de 12 meses. Mientras que Whiteley y et al en el 2013, proponen un tiempo promedio de intervención de 12 a 24 meses, por lo que se plantea que el apego a la dieta por el niño con TEA de nuestro estudio y sobre todo del cuidador fue de importancia para nuestros resultados.

Para la permeabilidad intestinal se observó una disminución en los signos y síntomas intestinales lo que sugiere que el consumo de los alimentos con gluten y caseína a menos de tres veces por semana (principalmente de pan de barra, yogurt y leche) mejora la capacidad de absorción de los nutrientes al presentar menor sintomatología intestinal

\section{Conclusiones}

Se concluye que una intervención nutricional a niños con TEA con dieta sin gluten y caseína se relaciona con el descenso de signos y síntomas gastrointestinales asociados a permeabilidad intestinal.

Dentro de las limitaciones del estudio tuvimos tiempo establecido de intervención, tamaño de la muestra de estudio, aun con estos inconvenientes se lograron obtener resultados significativos.

Se sugiere que se realice mayor investigación acerca del tema para en un futuro enriquecer y mejorar el tratamiento de los niños con TEA. 


\section{Bibliografía}

Adams B. J., Johansen J. L. Powell D. L. Quig D. and Rubin A. R. (2011). Gastrointestinal flora and gastrointestinal status in children with autism comparisons to typical children and correlation with autism severity. BCM Gastroenterology, 11-22.

Audisio, A., Laguzzi, J., Lavanda, I., Leal, M., Herrera, J., Carrazana, C., \& Pintos, C. C. (2013). Mejora de los síntomas del autismo y evaluación alimentaria nutricional luego de la realización de una dieta libre de gluten y caseína en un grupo de niños con autismo que acuden a una fundación. Nutrición clínica y dietética hospitalaria, 33(3), 39-47.

Cabanyes-Truffino J, García-Villamisar D. (2004) Identificación y diagnóstico precoz de los trastornos del espectro autista. Rev Neurol.;39(1):81-90

Elder, J. H., Shankar, M., Shuster, J., Theriaque, D., Burns, S., \& Sherrill, L. (2006). The gluten-free, casein-free diet in autism: results of a preliminary double blind clinical trial. Journal of autism and developmental disorders, 36(3), 413-420.

FAO/WHO/ONU. (2001). Human Energy Requirements. Food and nutrition technical report series. 20-32.

Ghalichi F. Ghaemmaghami J., Malek A., and Alireza O M. (2016). Effect of gluten free diet on gastrointestinal and behavioral indices for children with autism spectrum disorders: a randomized clinical trial. 2-7.

Gottschall E. (2004). Breaking the vicious cycle, intestinal health through diet.

Harris C. and Card B. (2012). A pilot study to evaluate nutritional influences on gastrointestinal symptoms and behavior patterns in children with autism spectrum disorder. Complementary therapies in medicine 20,437-440.

Horvath K. and Perman J. (2002). Austistic disorder and gastrointestinal disease. 14,583-587.

Lázaro C. P., Pondé M. P. and Rodríguez L. E. A. (2016). Opioid peptides and gastrointestinal symptoms in autism spectrum disorders. Revista Brasileira de Psiquiatría 6; 38:243-246.

Organización Mundial de la Salud. (2008).Curso de capacitación sobre la evaluación del crecimiento del niño. Ginebra.

Pedersen L. Parlar S., Kvist K, Whiteley P. and Shattock P. (2014) Data mining the ScanBrit Study of a glutenand casein- free dietary intervention for children with autism spectrum disorders: behavioral and

psychometric measures of dietary response. 5,207-213.

Pennesi M. C. and Cousino K. C. (2012). Effectiveness of the gluten-free, casein-free diet for children diagnosed with autism spectrum disorder: Based on parental report Nutritional Neuroscience. 15(2): 85-91.

Salles T. T. F. S., Boroni M. A. P., Silva S. N. C. Frias R. and Gouveia P. M. C. (2014) Intestinal permeability measurements: general aspects and possible pitfalls. Nutr Hosp. 29 (2): 269-281.

Toh C., M., and Allen V. E., (2015). The Microbiome in Autism Spectrum Disorder. The human gut microbiota with reference to autism spectrum disorder: considering the whole as more than a sum of its parts. Microbial Ecology in Health and Disease. 26.

Villatoro M., V., Rodríguez V. G., y Pardo P. A., (2015) Trasplante de microbiota fecal: revisión de la literatura y reporte del primer caso en el Hospital Central Militar. Rev. Sanid Milit Mex. 69, 242-248.

Whiteley P., Shattock P., Knivsberg A. M. Seim A., Reichelt K. L., Todd L., Carr K. and Hooper M., (2013). Gluten- and casein-free dietary intervention for autism spectrum conditions. Frontiers in Human Neuroscience.6, 344.

World Health Organization. (1995). Physical Status: The use and Interpretation of Anthropometry.161-255. 